\title{
Pathogen-Specific Clinical Trials: A New Paradigm in Clinical Trials for Multidrug-Resistant Organisms
}

\author{
Sarah C. J. Jorgensen · Michael J. Rybak
}

Received: September 13, 2018 / Published online: October 3, 2018

(C) The Author(s) 2018

Keywords: Carbapenem-resistant

Enterobacteriaceae; Meropenem-vaborbactam; TANGO-II

Multidrug-resistance (MDR) among Gram-negative bacteria represents an urgent public health threat that continues to escalate as these pathogens relentlessly spread and acquire new resistant determinants [1]. Carbapenems have traditionally fulfilled a critical role in the treatment of MDR infections. The global dissemination of beta-lactamases capable of hydrolyzing carbapenems has therefore forced clinicians to resort to last-line agents, such as the polymyxins, aminoglycosides and tigecycline, which lack robust efficacy data and carry the potential for significant toxicity [2]. Partly

Enhanced Digital Features To view enhanced digital features for this article go to https://doi.org/10.6084/ m9.figshare.7093964.

S. C. J. Jorgensen · M. J. Rybak ( $\square)$

Anti-infective Research Laboratory, Eugene Applebaum College of Pharmacy and Health Sciences, Wayne State University, Detroit, MI, USA e-mail: m.rybak@wayne.edu

\section{J. Rybak}

Department of Medicine, Division of Infectious Diseases, School of Medicine, Wayne State University, Detroit, MI, USA

M. J. Rybak

Detroit Medical Center, Detroit, MI, USA due to the paucity of safe and effective treatments, crude mortality rates approaching 50\% are often documented in patients infected with carbapenem-resistant Enterobacteriaceae (CRE) [3-6]. Developing novel antibiotics that remain stable against these enzymes has proved tremendously challenging for the pharmaceutical industry [1]. An alternative strategy, whereby the activity of an existing agent is restored by combining it with a novel beta-lactamase inhibitor, has, however, translated into initial progress in the battle against MDR pathogens. The first of these combinations to enter the clinic, ceftazidime-avibactam, demonstrated superior outcomes in comparison with older, more toxic regimens in small observational studies of patients with CRE infections $[7,8]$. However, suboptimal response in certain patient populations (i.e. those with pneumonia or receiving renal replacement therapy), as well the development of on-therapy resistance in up to $10 \%$ of patients, underscores the urgent need for continued antibiotic innovation $[9,10]$.

In this issue, Wunderink and colleagues report the results of a pathogen-specific, Phase III, multinational, open-label, randomized, controlled trial (TANGO II) comparing the novel carbapenem-beta-lactamase inhibitor, meropenem-vaborbactam, with best available therapy (BAT) for the treatment of serious CRE infections. Pathogen-specific clinical trials 
represent a new paradigm for antibactierial drug development [11]. In the case of infections caused by MDR pathogens, there is a critical need for novel therapies that are effective irrespective of the primary source of infection. Therefore, pathogen-specific trials may provide more clinically useful information than traditional clinical trials used to gain regulatory approval that focus on a specific infectious disease syndrome, generally in patients at low risk for antimicrobial resistance [11]. TANGO II included patients with CRE bloodstream infection, hospital-acquired or ventilator-associated pneumonia (HAP/VAP), complicated intra-abdominal infection or complicated urinary tract infection. The clear strengths of the trial, in addition to a pathogen focus, include the prospective design, randomization, stratification by infection type, inclusion of medically complicated patients, and blinded evaluation of outcomes by both the local site investigator and an adjudication committee. Among patients with a confirmed CRE infection, those assigned to meropenem-vaborbactam experienced higher rates of clinical cure, reduced all-cause mortality and lower nephrotoxicity versus those assigned to BAT.

Do these positive results finally establish an antibiotic as the preferred standard of care for CRE infections? Although the investigators are to be applauded for their meticulous execution of a clinical trial in a complex patient population, there are some limitations that need to be considered when drawing specific conclusions from these data. First, a total of only 77 patients were randomized, of whom 47 had a confirmed CRE infection. The present relatively low frequency of CRE infections provides a very small patient-pool from which clinical trials can recruit, and hence trials like TANGO II must be global, recruiting patients from countries with differing standards of care, approved comparator antibiotics, and regulatory frameworks. Furthermore, patients with serious CRE infections are often acutely ill, requiring intensive adjunctive care that may make obtaining consent for enrolment difficult $[3,11]$. Perhaps due to difficulties in consenting more critically ill patients, in the present study, less than $20 \%$ of patients required intensive care unit (ICU) admission and fewer than 50\% presented with the systemic inflammatory response syndrome (SIRS), suggesting overall a lower acuity of illness than is often seen in real-world practice, where approximately $50 \%$ of patients with CRE infections have required ICU care [7, 12]. Patients with CRE infections also typically carry a substantial burden of chronic comorbidity and immunocompromising conditions $[3,7,12]$. These characteristics were well represented in the TANGO II cohort, with over 50\% having a Charlson Comorbidity Index score above five and nearly one-third categorized as immunocompromised. This low baseline health status, however, portends a poor prognosis irrespective of antibiotic efficacy and complicates attempts to correlate crude and attributable mortality. Baseline and infectioninduced organ dysfunction is also relatively common in patients with MDR infections, leading to unpredictable and variable drug pharmacokinetics [3]. Although this may make target drug level attainment more challenging, inclusion of such patients in clinical trials provides valuable data on the effectiveness and safety of adjusted dosing strategies. It is disappointing then that only approximately $10 \%$ of patients in TANGO II had severe renal impairment (estimated creatinine clearance $<30 \mathrm{~mL} /$ $\mathrm{min}$ ) and that those requiring continuous renal replacement were excluded. Finally, the lack of a clear consensus in the preferred treatment strategy for CRE infections leaves no standard basis for comparison. This was illustrated by the ten different single agent or combination regimens used in the BAT arm. Although it may be argued that ceftazidime-avibactam has emerged as the drug of choice in many regions, it is also notable that this agent was administered to only one patient in the BAT group.

Despite these largely unavoidable limitations, the findings of the study do contain potentially valuable efficacy and safety insights. First, with regards to in vitro activity, although meropenem-vaborbactam minimum inhibitory concentrations (MICs) were not reported, 12\% $(5 / 41)$ of K. pneumoniae isolates were reported to show baseline meropenem-vaborbactam resistance (MIC $>4 / 8$ ). Four of these isolates produced metallo-beta-lactamases or class D 
carbapenemases (against which vaborbactam is known to be inactive [13]), while the remaining isolate produced KPC-3. Although rare, surveillance studies have documented pre-existing meropenem-vaborbactam resistance among KPC-producing $K$. pneumoniae, typically mediated by increased KPC expression and/or mutations to ompK35 and ompK36 [13, 14]. These findings attest to the critical need for rapid diagnostic tests that are capable of detecting both genetic markers of resistance and antimicrobial susceptibilities. Although it is important not to draw firm conclusions from the small number of isolates tested, it is encouraging that the specter of on-therapy was not raised in this study, with all follow-up cultures from patients in the meropenem-vaborbactam group $(n=32)$ maintaining susceptibility. One organism did develop a fourfold increase in MIC during treatment, but the follow-up MIC remained within the susceptible range $(1 \mathrm{mg} / \mathrm{L})$. It is reasonable to speculate that the stability of meropenem to extended-spectrum beta-lactamases and AmpC enzymes, together with preserved activity in high inoculum infections $[15,16]$, may provide a stronger antibiotic backbone compared to cephalosporins when co-formulated with carbapenemase inhibitors.

In terms of patient outcomes, the overall 28 -day mortality of $16 \%$ in the meropenemvaborbactam group does suggest progress in the battle against CRE, although it must be remembered that over one-third of patients had a low-risk urinary source of infection. Notably, there were no deaths among the four patients treated with meropenem-vaborbactam for CRE HAP/VAP; the one patient treated with BAT for HAP/VAP did, unfortunately, pass away. Perhaps most impressively, among patients who did not experience antibiotic failure prior to meropenem-vaborbactam initiation $(n=23)$, there was only one death. This finding underscores the importance of early effective therapy in serious CRE infections and again points to the critical role of rapid diagnostics in optimizing the use of new agents. Whereas combination therapy has been the standard for CRE infections [3, 5], even following the introduction of ceftazidime-avibactam $[10,17,18]$, these outcomes were achieved with meropenemvaborbactam monotherapy which, in addition, demonstrated a favorable tolerability profile, particularly with respect to renal adverse effects.

The outcomes in certain high-risk patient subgroups, however, are a sobering reminder that there is still much room for improvement in the management of CRE infections. In patients with positive blood culture, SIRS or age $\geq 65$ years, 28-day mortality rates ranged between 26 and $29 \%$ in the meropenemvaborbactam group (vs. $38-66 \%$ in the BAT arm). Perhaps most alarming was that only 2 of 7 meropenem-vaborbactam patients with creatinine clearance $<50 \mathrm{~mL} / \mathrm{min}$ achieved clinical success, and mortality in this subgroup was over $40 \%$.

To adapt to the unmet medical need caused by infections due to the MDR pathogens, we must advance the paradigm of antimicrobial precision medicine. To this end, innovative, pathogen-focused trials enrolling vulnerable patients are essential to inform on the efficacy and safety of novel agents in their target populations. TANGO II has provided clinically useful insights into the safety and efficacy of meropenem-vaborbactam in patients with CRE infections. Moving forward, it will be important for the infectious diseases community to share their experiences with meropenem-vaborbactam so that additional insight can be gained into important therapeutic questions not addressed in this study, such as associated collateral damage, emergence of resistance with more widespread use, and the role, if any, of combination meropenem-vaborbactam therapy.

\section{ACKNOWLEDGEMENTS}

Funding. No funding or sponsorship was received for the writing or publication of this editorial.

Authorship. All named authors meet the International Committee of Medical Journal Editors (ICMJE) criteria for authorship for this article, take responsibility for the integrity of 
the work as a whole, and have given their approval for this version to be published.

Disclosures. Michael J. Rybak has received research support, has consulted or has been a speaker for Achaogen, Allergan, Bayer, Melinta, Merck, Motif, Nabriva, Shionogi, Tetraphase and Theravance. Sarah C. J. Jorgensen has nothing to disclose.

Compliance with Ethics Guidelines. This editorial is based on previously conducted studies and does not contain any studies with human participants or animals performed by any of the authors.

Open Access. This article is distributed under the terms of the Creative Commons Attribution-NonCommercial 4.0 International License (http://creativecommons.org/licenses/ by-nc/4.0/), which permits any noncommercial use, distribution, and reproduction in any medium, provided you give appropriate credit to the original author(s) and the source, provide a link to the Creative Commons license, and indicate if changes were made.

\section{REFERENCES}

1. Doi Y, Bonomo RA, Hooper DC, Kaye KS, Johnson JR, Clancy CJ, et al. Gram-negative bacterial infections: research priorities, accomplishments, and future directions of the antibacterial resistance leadership group. Clin Infect Dis. 2017;64(suppl_1): S30-5.

2. Wenzler E, Goff DA, Humphries R, Goldstein EJC. Anticipating the unpredictable: a review of antimicrobial stewardship and acinetobacter infections. Infect Dis Ther. 2017;6(2):149-72.

3. Alexander EL, Loutit J, Tumbarello M, Wunderink R, Felton T, Daikos G, et al. Carbapenem-resistant enterobacteriaceae infections: results from a retrospective series and implications for the design of prospective clinical trials. Open Forum Infect Dis. 2017;4(2):ofx063.

4. Balandin Moreno B, Fernandez Simon I, Pintado Garcia V, Sanchez Romero I, Isidoro Fernandez B, Romera Ortega MA, et al. Tigecycline therapy for infections due to carbapenemase-producing
Klebsiella pneumoniae in critically ill patients. Scand J Infect Dis. 2014;46(3):175-80.

5. Daikos GL, Tsaousi S, Tzouvelekis LS, Anyfantis I, Psichogiou M, Argyropoulou A, et al. Carbapenemase-producing Klebsiella pneumoniae bloodstream infections: lowering mortality by antibiotic combination schemes and the role of carbapenems. Antimicrob Agents Chemother. 2014;58(4):2322-8.

6. Tumbarello M, Viale P, Viscoli C, Trecarichi EM, Tumietto F, Marchese A, et al. Predictors of mortality in bloodstream infections caused by Klebsiella pneumoniae carbapenemase-producing K. pneumoniae: importance of combination therapy. Clin Infect Dis. 2012;55(7):943-50.

7. van Duin D, Lok JJ, Earley M, Cober E, Richter SS, Perez F, et al. Colistin versus ceftazidime-avibactam in the treatment of infections due to carbapenemresistant Enterobacteriaceae. Clin Infect Dis. 2018;66(2):163-71.

8. Shields RK, Nguyen MH, Chen L, Press EG, Potoski $\mathrm{BA}$, Marini RV, et al. Ceftazidime-avibactam is superior to other treatment regimens against carbapenem-resistant Klebsiella pneumoniae bacteremia. Antimicrob Agents Chemother. 2017; 61(8):e00883-17.https://doi.org/10.1128/AAC.0088317.

9. Shields RK, Nguyen MH, Chen L, Press EG, Kreiswirth BN, Clancy CJ. Pneumonia and renal replacement therapy are risk factors for ceftazidimeavibactam treatment failures and resistance among patients with carbapenem-resistant Enterobacteriaceae infections. Antimicrob Agents Chemother. 2018;62(5):e02497-17. https://doi.org/10.1128/ AAC.02497-17.

10. Shields RK, Potoski BA, Haidar G, Hao B, Doi Y, Chen L, et al. Clinical outcomes, drug toxicity, and emergence of ceftazidime-avibactam resistance among patients treated for carbapenem-resistant Enterobacteriaceae infections. Clin Infect Dis. 2016;63(12):1615-8.

11. Infectious Diseases Society of A. White paper: recommendations on the conduct of superiority and organism-specific clinical trials of antibacterial agents for the treatment of infections caused by drug-resistant bacterial pathogens. Clin Infect Dis. 2012;55(8):1031-46.

12. King M, Heil E, Kuriakose S, Bias T, Huang V, ElBeyrouty C, et al. Multicenter study of outcomes with ceftazidime-avibactam in patients with carbapenem-resistant Enterobacteriaceae infections. Antimicrob Agents Chemother. 2018;61(7):e00 449-17. https://doi.org/10.1128/AAC.00449-17. 
13. Jorgensen SCJ, Rybak MJ. Meropenem and vaborbactam: stepping up the battle against carbapenemresistant Enterobacteriaceae. Pharmacotherapy. 2018;38(4):444-61.

14. Sun D, Rubio-Aparicio D, Nelson K, Dudley MN, Lomovskaya O. Meropenem-vaborbactam resistance selection, resistance prevention, and molecular mechanisms in mutants of KPC-producing Klebsiella pneumoniae. Antimicrob Agents Chemother. 2017;61(12):e01694-17. https://doi.org/ 10.1128/AAC.01694-17.

15. Martinez-Martinez L, Pascual A, Hernandez-Alles S, Alvarez-Diaz D, Suarez AI, Tran J, et al. Roles of beta-lactamases and porins in activities of carbapenems and cephalosporins against Klebsiella pneumoniae. Antimicrob Agents Chemother. 1999; 43(7):1669-73.
16. Moellering RC Jr, Eliopoulos GM, Sentochnik DE. The carbapenems: new broad spectrum beta-lactam antibiotics. J Antimicrob Chemother. 1989; 24(Suppl A):1-7.

17. Temkin E, Torre-Cisneros J, Beovic B, Benito N, Giannella M, Gilarranz R, et al. Ceftazidime-avibactam as salvage therapy for infections caused by carbapenem-resistant organisms. Antimicrob Agents Chemother. 2017;61(2): e01964-16. https:// doi.org/10.1128/AAC.01964-16.

18. Tumbarello M, Trecarichi EM, Corona A, De Rosa FG, Bassetti M, Mussini C, et al. Efficacy of ceftazidime-avibactam salvage therapy in patients with infections caused by KPC-producing Klebsiella pneumoniae. Clin Infect Dis. 2018. https://doi.org/ 10.1093/cid/ciy492. 